\title{
OLIGODENDROGLIOMAS
}

\section{ESTUDO ANATOMOPATOLÓGICO E CLÍNICO DE 15 CASOS}

\author{
JORGE SERGIO REIS FILHO*, MÁRIO RODRIGUES MONTEMÓR NETTO*, \\ BEATRIZ GARCIA SLUMINSKY**, LINEI AUGUSTA BROLINI DELLÉ**, \\ AFONSO ANTONIUK ${ }^{* * *}$, RICARDO RAMINA ${ }^{* * * *}$, LUIZ FERNANDO BLEGGI TORRES ${ }^{* * * * *}$
}

\begin{abstract}
RESUMO - Oligodendrogliomas correspondem a 4-5\% dos tumores primários do sistema nervoso central apresentando crescimento infiltrativo e lento. Relatamos os achados anatomopatológicos e clínicos de 15 casos de oligodendrogliomas. Oito pacientes eram do sexo masculino e 7 do feminino. As idades oscilaram entre $17 \mathrm{e}$ 66 anos, apresentando média de 39,73 anos. A sintomatologia apresentada correspondeu ao crescimento expansivo, sendo cefaléia $(60 \%)$ e crises convulsivas $(60 \%)$ os sintomas mais frequentes. O lobo frontal $(\mathrm{n}=6)$ foi o sítio anatômico mais acometido, seguido pelo parietal $(n=2)$, temporal $(n=1)$ e occipital $(n=1)$. Cinco pacientes foram submetidos a ressecção total do tumor e 10 pacientes a exerese parcial; dentre estes, 3 foram submetidos a radioterapia adjuvante, 1 a quimioterapia e 1 a quimio e radioterapia. Evidenciou-se taxa de recidiva tumoral total de $60 \%$ em período médio de 32 meses de acompanhamento. Cinco recidivas tumorais ocorreram nos pacientes submetidos apenas ao tratamento cirúrgico e quatro nos pacientes submetidos a quimio ou radioterapia adjuvante. Estes achados aproximam-se dos encontrados na literatura, auxiliando na compreensão do comportamento biológico deste raro tumor cerebral.
\end{abstract}

PALAVRAS-CHAVE: oligodendrogliomas, gliomas, sistema nervoso central.

\section{Oligodendroglioma: a pathological and clinical study of 15 cases}

ABSTRACT - Oligodendrogliomas account for $4-5 \%$ of primary central nervous system tumours with a slow and infiltrative growth. We report the clinical and pathological findings of 15 cases of oligodendrogliomas. Eight patients were males and 7 were females. The ages ranged between 17 and 66 years, with a mean of 39.73 years. The symptoms reflected the growth and topography of the tumours; migraine $(60 \%)$ and seizures $(60 \%)$ were the most frequent symptoms. Frontal $(n=6)$, parietal $(n=2)$, temporal $(n=1)$ and occipital $(n=1)$ lobes were affected. Five patients undergone total resection of the tumor and 10 were submitted to partial resection, from which 3 received adjuvant radiotherapy, 1 adjuvant chemotherapy and 1 chemotherapy and radiotherapy. The overall recurrence rate was $60 \%$ for a 32 month follow up. Five recurrences were observed in patients submitted only to the surgical treatment and 4 in which adjuvant radio or chemotherapy were performed. These results are similar with the literature and may contribute to further understanding the biological behavior of these rare tumours.

KEYWORDS: oligodendroglioma, gliomas, central nervous system.

Oligodendrogliomas são raros tumores encefálicos primários compostos por células que morfologicamente se assemelham a oligodendrócitos ${ }^{1-3}$, descritos inicialmente por Bailey \& Cushing ${ }^{4}$. Apesar de sua histogênese incerta, apresentam um curso indolente e bom prognóstico quando comparados a outras neoplasias gliais ${ }^{1,5-8}$. Relatamos os achados anatomopatológicos e clínicos de

Estudo realizado na Seção de Microscopia Eletrônica e Neuropatologia do Serviço de Anatomia Patológica (SAP) do Hospital de Clínicas (HC) da Universidade Federal do Paraná (UFPR), Curitiba, PR.: *Médico Residente em Anatomia Patológica - SAP - HC- UFPR; **Estagiário da Seção de Microscopia Eletrônica e Neuropatologia - SAP - HC - UFPR; ***Professor Titular da Disciplina de Neurocirurgia UFPR; ****Médico Neurocirurgião do Hospital das Nações - Curitiba;*****PhD em Neuropatologia (Londres), Chefe do SAP - HC - UFPR. Aceite: 17-dezembro-1998.

Dr. Jorge Sergio Reis Filho - Serviço de Anatomia Patológica, Hospital de Clínicas - Rua General Carneiro 18180060-900 Curitiba PR - Brasil. Fax 041264 1304. E-mails: jsreis@ hotmail.com ou neuropat@hc.ufpr.br 
15 casos de oligodendrogliomas diagnosticados em pacientes da Cidade de Curitiba no período compreendido entre 1990 e 1997, realizando correlação entre o tratamento realizado e o prognóstico dos portadores desta neoplasia. Até o presente momento, este é o primeiro estudo clínico-patológico na literatura brasileira indexada no MEDLINE e LILACS.

\section{MÉTODO}

Este estudo deriva da linha de pesquisa denominada "Banco de Patologia Tumoral do Sistema Nervoso Central da População da Cidade de Curitiba", que tem como objetivo tabular todos os dados referentes aos tumores do sistema nervoso central (SNC) que acometem a população dessa cidade e sua região metropolitana. A pesquisa tem o intuito de analisar epidemiologicamente os principais tumores do SNC. Para tanto, foram pesquisados os livros de registros ou arquivos de biópsias do período de 1990 a 1997 dos Serviços de Anatomia Patológica dos principais hospitais da Cidade de Curitiba que são responsáveis por mais de $95 \%$ da rotina neurocirúrgica do município. Do total de biópsias de diversos órgãos e tecidos realizadas neste período, foram compiladas as de SNC, pesquisando-se o sexo, idade, localização tumoral e diagnóstico histológico. Deste total, foram separados os oligodendrogliomas avaliando-se as variáveis sexo, idade e localização tumoral. Os dados clínicos dos pacientes, como história clínica, tempo de evolução dos sintomas, exame físico, tratamento e evolução foram obtidos a partir dos prontuários médicos. Todas as biópsias foram preparadas conforme técnicas histológicas convencionais ${ }^{9}$, sendo utilizados os critérios da Classificação da Organização Mundial de Saúde(OMS) ${ }^{3}$ para o diagnóstico dos oligodendrogliomas.

\section{RESULTADOS}

Das 3318 biópsias realizadas, 2427 (73,14\%) corresponderam a neoplasias primárias do SNC e, dentre estas, 38 (1,56\%) tiveram diagnóstico histológico de oligodendrogliomas (grau II). Destes, 23 não apresentaram dados clínicos e prognósticos suficientes para a inclusão no estudo. Dos quinze pacientes analisados, 8 ocorreram em pacientes do sexo feminino. As idades variaram de 14 a 66 anos, com média de 39,73 anos (Fig 1). Quanto à localização, treze casos acometeram hemisférios cerebrais, sendo 7 no hemisfério esquerdo e seis no direito; dois casos eram intraventriculares (Fig 2). Os sinais e sintomas observados incluiram cefaléia (60\% dos pacientes), crises convulsivas (60\% dos pacientes), sinais de hipertensão endocraniana e desordens neurológicas focais (Fig 3), com período de evolução variando de 2 a 36 meses, com uma média de 12,93 meses. Cinco pacientes foram submetidos a ressecção cirúrgica total , 5 a exérese parcial, 3 a exérese parcial associada a radioterapia adjuvante, 1 a ressecção parcial seguida de radio e quimioterapia e 1 a tumorectomia associada a quimioterapia (Fig 4). Evidenciouse taxa de recidiva tumoral total de $60 \%$, sendo $50 \%$ nos pacientes submetidos ao tratamento cirúrgico parcial ou total com período médio (PM) entre o tratamento e a recidiva de 38,4 meses, 66,6\% nos que receberam radioterapia adjuvante $(\mathrm{PM}=18)$ e $100 \%$ nos dois pacientes que receberam quimioterapia associada (PM = 30 meses) (Tabela 1). Apenas um caso de progressão maligna fora observado 6 anos após o tratamento cirúrgico.

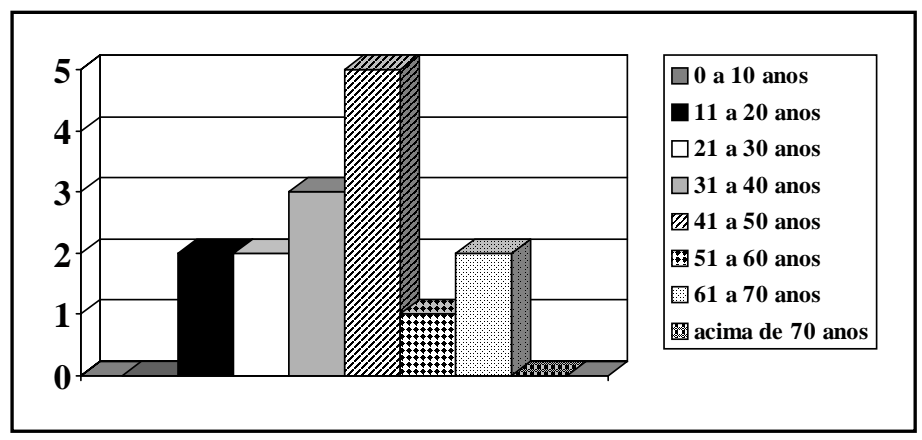

Fig 1. Distribuição dos pacientes portadores de oligodendrogliomas quanto à faixa etária. 


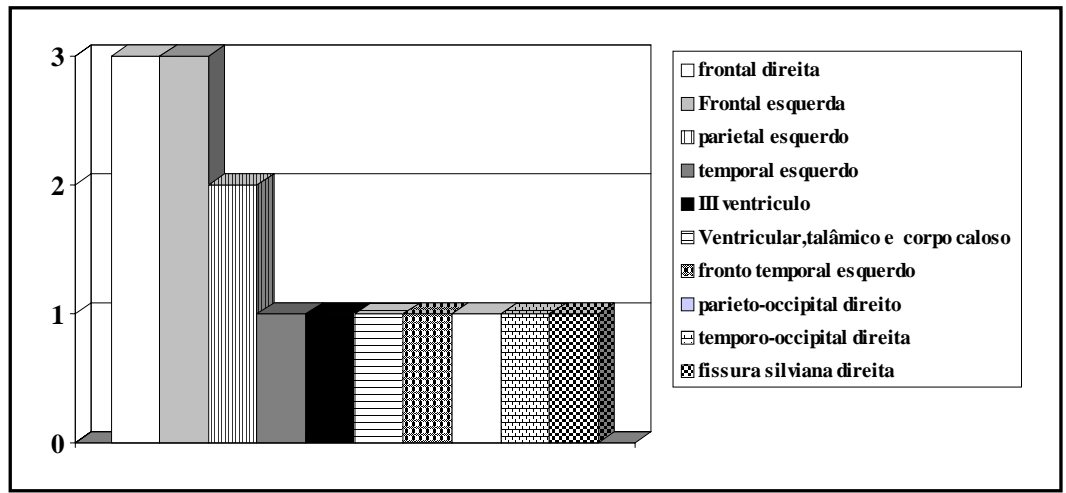

Fig 2. Distribuição dos casos de oligodendroglioma de acordo com a localização tumoral.

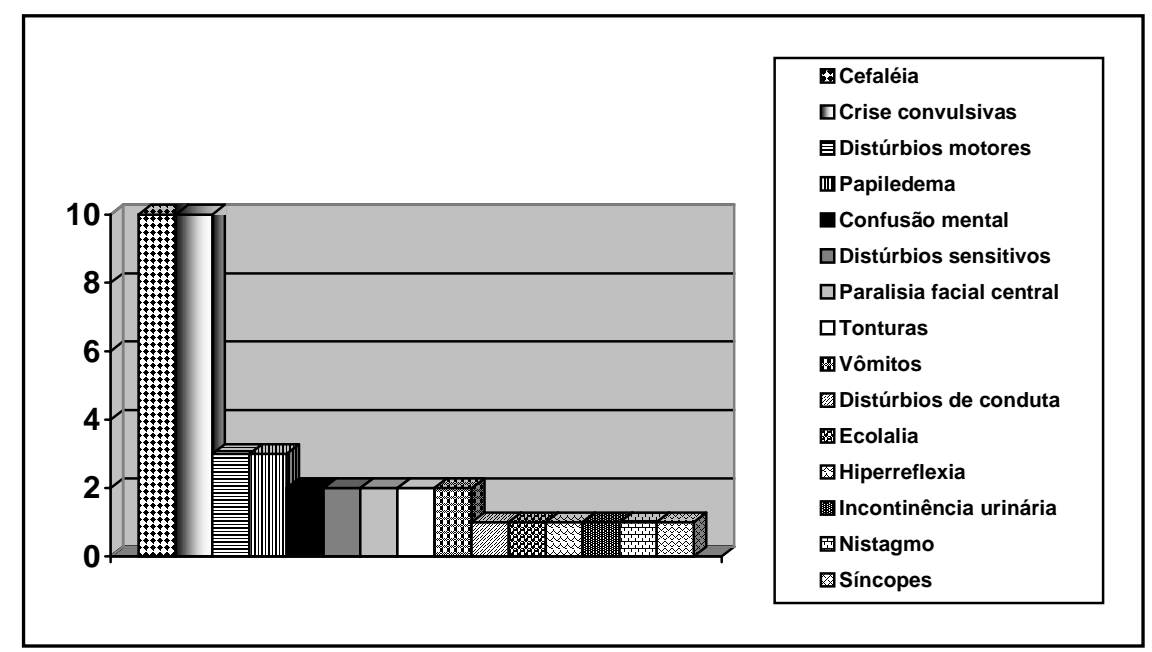

Fig 3. Sintomas apresentados pelo pacientes portadores de oligodendroglioma.

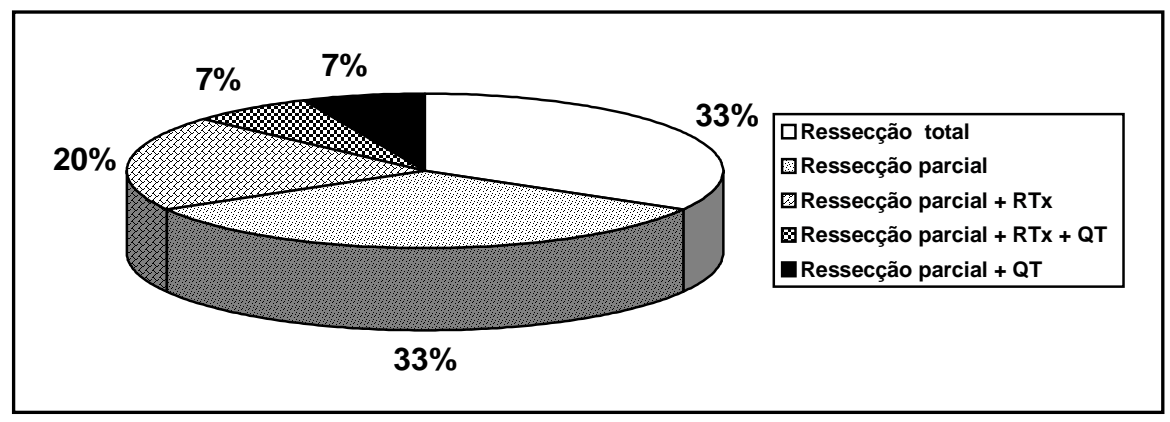

Fig 4. Tratamento dos pacientes portadores de oligodendroglioma.

$R T x$, radioterapia adjuvante; $Q T$, quimioterapia adjuvante. 
Tabela 1. Tabela demonstrando as taxas de recidiva tumoral nos pacientes portadores de oligodendrogliomas.

\begin{tabular}{lccc}
\hline Tratamento & Recidiva & $\%$ & $\begin{array}{c}\text { Período médio entre o tratamento } \\
\text { e a recidiva (meses) }\end{array}$ \\
\hline Ressecção total & $3 / 5$ & 60 & 32 \\
Ressecção parcial & $2 / 5$ & 40 & 48 \\
Ressecção parcial + RTx & $2 / 3$ & 66,6 & 18 \\
Ressecção parcial + QTx & $1 / 1$ & 100 & 36 \\
Ressecção parcial + RTx + Qt & $1 / 1$ & 100 & 24 \\
Total & $9 / 15$ & 60 & 32 \\
\hline
\end{tabular}

RTx, radioterapia; QTx, quimioterapia.

\section{DISCUSSÃO}

Os oligodendrogliomas são neoplasias relativamente raras, correspondendo a cerca de 4 a $5 \%$ de todos os tumores cerebrais primários ${ }^{8}$. Acometem predominantemente adultos, com a moda da incidência entre a quinta e sexta décadas de vida, manifestando-se na proporção de 2:1 entre homens e mulheres ${ }^{2,7,8}$. Na presente série, os oligodendrogliomas representaram 1,56\% das neoplasias primárias do SNC. Cerca de $6 \%$ dos oligodendrogliomas acometem pacientes pediátricos, com os sintomas manifestando-se até 10 anos nos tumores supratentoriais ${ }^{8}$ e 7,5 anos nos pacientes acometidos por tumores infratentoriais ${ }^{10}$. Não mais que 10 casos de ocorrência familial de oligodendrogliomas foram relatados e a associação de oligodendrogliomas com síndromes tumorais familiais não é comprovada. Em nossa casuística houve maior prevalência de casos na quinta década, entretanto com leve predomínio em mulheres.

Os oligodendrogliomas originam-se preferencialmente na substância branca dos hemisférios cerebrais, sendo únicos na sua grande maioria. O lobo frontal está acometido em cerca de 50 a $65 \%$ dos pacientes. Os lobos temporal, parietal e occipital são progressivamente menos afetados. Ventrículos, cerebelo, ponte, medula e meninges raramente são acometidos ${ }^{1,2,7,8,11}$. Em nossa casuística, $86,6 \%(n=13)$ dos tumores localizavam-se em hemisférios cerebrais, sendo que $40 \%(n=6)$ ocorreram em lobo frontal. Os dois casos intraventriculares receberam especial atenção no diagnóstico diferencial com neurocitomas centrais, porém a imuno-histoquímica para marcadores neuronais (sinaptofisina e enolase neurônio específica) foi negativa, corroborando o diagnóstico de oligodendroglioma.

A sintomatologia apresentada pelos pacientes com oligodendrogliomas é decorrente da localização e do crescimento infiltrativo do tumor, sendo constituída de distúrbios epileptiformes e cefaléia com longo período de evolução, o qual pode exceder $5 \operatorname{anos}^{2}$. Em nosso estudo, os principais sintomas foram cefaléia $(66,6 \%)$ e crises convulsivas $(66,6 \%)$, apresentando tempo de evolução médio de 12,92 meses. Síndrome de hipertensão endocraniana e desordens neurológicas focais são possíveis manifestações ${ }^{4}$, entretanto, menos frequentes, o que ocorreu em nossa casuística.

À análise neurorradiológica, as massas tumorais podem ser observadas tanto pela RNM quanto pela TAC sendo caracterizados por massas lobulares heterogêneas e bem delimitadas ${ }^{12}$. Calcificação tipo serpinginosa é achado frequente, bem como o edema peritumoral. Em alguns casos podem ser encontradas hemorragias intra-neoplásicas e degenerações císticas ${ }^{12}$.

O exame histopatológico evidencia aspecto monomórfico com moderada celularidade ${ }^{1-3,7,8,13}$. Áreas de maior celularidade podem ocorrer no centro de nódulos circunscritos por áreas hipocelulares ${ }^{1-}$ 3,7,8,13. As células neoplásicas são arredondadas, com citoplasma claro, com limites bem definidos, 


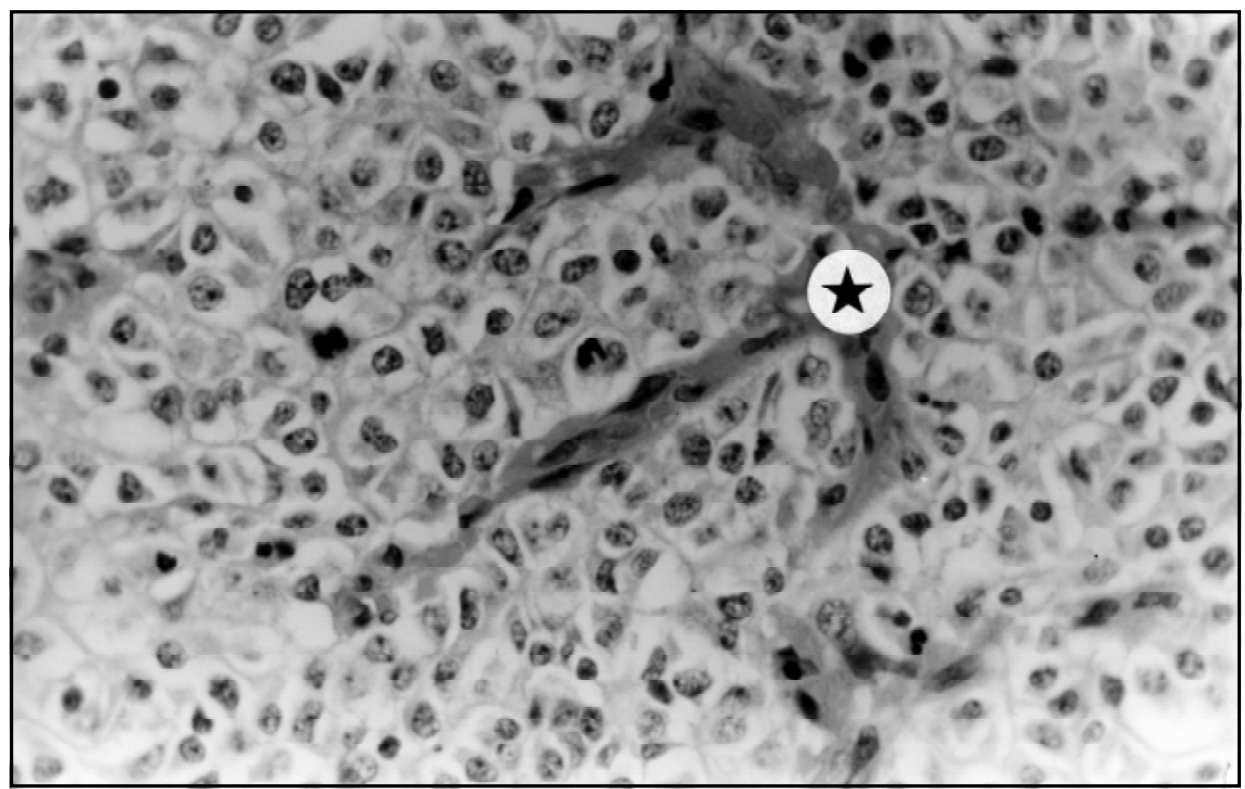

Fig. 5. Corte histológico de oligodendroglioma composto por células relativamente uniformes, citoplasma claro com limites celulares nítidos. Presença de proliferação vascular capilar arborescente (*). HE x 400.

apresentando núcleos arredondados, discretamente maiores e com cromatina mais densa quando comparados com os oligodendrócitos normais ${ }^{1-3,7,8,13}$. A atividade mitótica é baixa, não sendo evidenciadas mitoses atípicas ${ }^{1-3,7,8,13}$. O achado de aglomerados celulares em "favo-de-mel", apesar de nem sempre presente, pode direcionar o diagnóstico. A presença de microcalcificações é frequente, ocorrendo na periferia do tumor ou no córtex adjacente. Áreas com deposição de mucina extracelular, microcistificação e pseudorosetas não são infrequentes. Os tumores exibem tendência a apresentarem hemorragias intratumorais. Em alguns casos o estroma capilar arborescente pode dividir o tumor em lóbulos ${ }^{1-3,7,8,13}$ (Fig 5).

O crescimento dos oligodendrogliomas é difuso na substância branca e infiltrativo no córtex, onde se formam estruturas como satelitose perineuronal, agregados perivasculares e acúmulos subpiais. A infiltração leptomeníngea pode acarretar reação desmoplásica e fibrótica ${ }^{1-3,7,8,13}$.

A análise imuno-histoquímica ainda não determinou um marcador biológico específico para células oligodendrogliais neoplásicas, podendo haver positividade para proteína S-100, Leu-7 e vimentina $^{14-16}$. Proteína glial fibrilar ácida pode ser reativa em oligodendróctios minigemistocísticos ou gliofibrilares, o que é corroborado por estudos ultraestruturais ${ }^{15}$. A utilização de marcadores de proliferação celular, como Ki67(MIB-1) evidenciou-se significativamente positiva em oligodendrócitos gliofibrilares, mas pouco frequente no restante das células neoplásicas, o que enfatiza o crescimento lento do tumor ${ }^{5,6}$.

Os pacientes portadores de oligodendrogliomas (grau II da OMS) apresentam sobrevida média

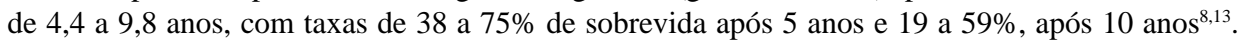
Recidiva local é frequente, porém a progressão maligna é rara ${ }^{1,2,5,7,8,13}$. Em nosso estudo, recidiva tumoral ocorreu em $50 \%$ dos casos submetidos apenas a tratamento cirúrgico, 66,6\% dos casos em que se associou radioterapia e em $100 \%$ dos casos em que se realizou quimioterapia adjuvante. Os fatores clínicos de bom prognóstico incluem baixa idade, localização em lobo frontal e possibilidade de tumorectomia total ${ }^{8,13}$, como corroborado pelo presente estudo. 


\section{REFERÊNCIAS}

1. Burger PC, Rawlings CE, Cox EB, McLendon RE, Schold SC Jr, Bullard DE. Clinicopathologic correlations in the oligodendroglioma. Cancer 1987;59:1345-1352

2. Chin HW, Hazel JJ, Kim TH, Webster JH. Oligodendroglioma: I. A clinical study of cerebral oligodendrogliomas. Cancer, 1980;45:1458-1466.

3. Kleihues P, Burger PC, Scheithauer BW. The new WHO classification of brain tumours. Brain Pathol, 1993;3:255-268.

4. Bailey P, Cushing H. A classification of tumours of the glioma group on a histogenetic basis with a correlation study of prognosis. Lippincott:Philadelphia, 1926.

5. Ganju V, Jenkins RB, O'Fallon JR, et al.. Prognostic factors in the gliomas: a multivariate analysis of clinical, pathologic, flow cytometric, cytogenetic, and molecular markers. Cancer, 1994;74:920-927.

6. Kros JM, Hop WC, Godschalk JJ, Krishnadath KK. Prognostic value of the proliferation-related antigen Ki-67 in oligodendrogliomas. Cancer, 1996;78:1107-1113.

7. Kros JM, Pieterman H, Van Eden CG, Avezaat CJ. Oligodendroglioma: The Rotterdan-Dijokzigt experience. Neurosurgery, 1994;34:959-966.

8. Mork SJ, Lindegaard KF, Halvorsen TB, et al. Oligodendroglioma: incidence and biological behaviour in a defined population. J Neurosurg, 1985;63:881-889.

9. Bancroft JD, Stevens A. Theory and practice of histological techniques. 2Ed. New York: Churchill Livingstone, 1982.

10. Packer RJ, Sutton LN, Rorke LB, et al.. Oligodendroglioma of the posterior fossa in childhood. Cancer, 1985;56:195-199.

11. Kros JM, Godschalk JJ, Krishnadath KK, Van Eden CG. Expression of p53 in oligodendrogliomas. J Pathol, 1993;171:285-290.

12. Lee Y, Van Tassel P. Intracranial oligodendrogliomas: imaging findings in 35 untreated cases. Am J Roentgenol, 1989;152:361-369.

13. Shaw EG, Scheithauer BW, O'Fallon JR, Tazeelar HD, Davis DH. Oligodendroglioma: the Mayo Clinic experience. J Neurosurg, 1992;76:428-434.

14. Kros JM, de Jong A A, van der Kwast TH. Ultrastructural characterization of transitional cells in oligodendrogliomas. J Neuropathol Exp Neurol, 1992;51:186-193.

15. Kros JM, Van Eden CG, Stefanko SZ, Waayer Van Batenburg M, van der Kwast TH. Prognostic implications of glial fibrillary acidic protein containing cell types in oligodendrogliomas. Cancer, 1990;66:1204-1212.

16. Sung CC, Collins R, Li J, et al.. Glycolipids and myelin proteins in human oligodendrogliomas. Glycoconj J, 1996;13:433-443. 